\title{
Evaluation of the stroke volume variation index as a predictor of fluid responsiveness during orthotopic liver transplantation.
}

\author{
André P. Schmidt, Mateus G. S. Tessmer, Elaine A. Felix. Jose Otavio C Auler Jr. \\ Department of Anaesthesia and Perioperative Medicine, Hospital de Clinicas de Porto Alegre, \\ Federal University of Rio Grande do Sul, Porto Alegre, Brazil
}

\section{Introduction:}

The major goal of fluid therapy is to optimize tissue perfusion, which is usually achieved by an increase in cardiac output $(1,2)$. The stroke volume variation (SVV) index has been shown to be a reliable predictor of fluid responsiveness in a variety of clinical settings $(1,2)$. However, it has not been extensively evaluated in the setting of orthotopic liver transplantation (OLT). The fluid therapy in cirrhotic patients undergoing orthotopic liver transplantation may be challenging and it is still controversial. Our main aim was to assess whether the SVV measured by the Flotrac/Vigileo ${ }^{\text {TM }}$ system can predict fluid responsiveness in patients submitted to OLT.

\section{Methods:}

Twenty-five (25) mechanically ventilated patients undergoing liver transplantation who needed volume expansion were included. All patients were monitored with pulmonary artery catheter, which measured the cardiac output on a continuous basis and the Flotrac/Vigileo ${ }^{\mathrm{TM}}$ system. A fluid challenge with $10 \mathrm{ml} / \mathrm{kg}$ ideal weight of albumin $2 \%$ was attempted during the following stages of surgery: hepatectomy $\left(T_{1}\right)$, anhepatic phase $\left(T_{2}\right)$, up to 30 min after venous reperfusion $\left(\mathrm{T}_{3}\right)$, up to 30 min after hepatic artery reperfusion $\left(\mathrm{T}_{4}\right)$, and at the beginning of abdominal closure $\left(\mathrm{T}_{5}\right)$. Central venous pressure (CVP), and pulmonary artery occlusion pressure (PAOP) and SVV were measured immediately before and up to $5 \mathrm{~min}$ after fluid challenge. Cardiac index $(\mathrm{Cl})$, measured by pulmonary artery catheter, was used to define responder patients if $\mathrm{Cl}$ increased by $15 \%$ or more after fluid challenge, otherwise, it was considered non-responsive. SVV and all other parameters were recorded at baseline and $5 \mathrm{~min}$ after the FC.

Table 1: Patient characteristics. Data are expressed as mean (SD) and absolute numbers

\begin{tabular}{lc}
\hline Variable & Value \\
\hline Age (years) & $55.2(6.1)$ \\
Gender $(\mathrm{M} / \mathrm{F})$ & $18 / 7$ \\
$\mathrm{BMI}^{*}\left(\mathrm{Kg} / \mathrm{m}^{2}\right)$ & $30.9(7.2)$ \\
Liver disease: & \\
$\quad$ Hepatitis C & 17 \\
Hepatitis B & 2 \\
Alcoholic & 5 \\
$\quad$ Cryptogenic & 1 \\
Child status: & \\
Child A & 2 \\
Child B & 8 \\
Child C & 15
\end{tabular}

*BMI: body mass index.

\section{Results:}

Forty-four fluid challenges were performed, with 30 (68.2\%) classified as responsive and 14 (3I.8\%) as nonresponsive. Receiver operating characteristic (ROC) analysis showed that SVV could be used to predict fluid responsiveness during OLT (AUC: 0.79I; $P<0.01$ ).

Table 2: Comparison of baseline haemodynamic data between groups. Data are expressed as mean (SD). SVV: stroke volume variation; PAOP: pulmonary artery occlusion pressure; CVP: central venous pressure; MAP: mean arterial pressure; $\mathrm{Cl}$ : cardiac index.

Fluid challenges $(n=44)$

\begin{tabular}{lccc}
\hline & $\mathrm{R}$ & $\mathrm{NR}$ & $P$ \\
& $(\mathrm{n}=30)$ & $(\mathrm{n}=14)$ & \\
$\mathrm{SVV}(\%)$ & $19,4(8,0)$ & $14,2(5,5)$ & $0,03^{*}$ \\
$\mathrm{PAOP}(\mathrm{mmHg})$ & $11,9(3,9)$ & $11,1(3,3)$ & 0,50 \\
$\mathrm{CVP}(\mathrm{mmHg})$ & $11,5(3,1)$ & $9,9(3,6)$ & 0.14 \\
$\mathrm{MAP}(\mathrm{mmHg})$ & $70,6(12,4)$ & $68,9(15,8)$ & 0,70 \\
$\mathrm{Cl}\left(\mathrm{l} / \mathrm{min} / \mathrm{m}^{2}\right)$ & $3,03(1,3)$ & $4,0(1,4)$ & $0,02^{*}$ \\
\hline
\end{tabular}

R- Responder; NR- Non-responder; ${ }^{*} \mathrm{P}<0,05$

Table 3: Comparison of the baseline hemodynamic data between groups at each individual phase. Data are expressed as mean (SD). SVV: stroke volume variation; PAOP: pulmonary artery occlusion pressure; CVP: central venous pressure; MAP: mean arterial pressure; $\mathrm{Cl}$ : cardiac index

Hepatectomy (Phase 1) $\quad$ Anhepatic phase (Phase 2) Post-reperfusion (Phases 3 to 5)

\begin{tabular}{lccccccccc}
\hline & $\mathrm{R}$ & $\mathrm{NR}$ & $P$ & $\mathrm{R}$ & $\mathrm{NR}$ & $P$ & $\mathrm{R}$ & $\mathrm{NR}$ & $P$ \\
& $(\mathrm{n}=12)$ & $(\mathrm{n}=6)$ & & $(\mathrm{n}=6)$ & $(\mathrm{n}=4)$ & & $(\mathrm{n}=12)$ & $(\mathrm{n}=4)$ & \\
$\mathrm{SVV}(\%)$ & $19,1(6,7)$ & $14,1(5,2)$ & $0,03^{*}$ & $19,3(9,0)$ & $16,0(6,9)$ & 0,59 & $20,2(7,8)$ & $13,5(4,7)$ & 0,07 \\
$\mathrm{PAOP}(\mathrm{mmHg})$ & $13,3(4,2)$ & $11,8(3,2)$ & 0,28 & $9,5(1,2)$ & $7,2(1,4)$ & 0,05 & $9,9(5,9)$ & $12,8(6,7)$ & 0,76 \\
$\mathrm{CVP}(\mathrm{mmHg})$ & $11,3(3,4)$ & $9,3(2,3)$ & 0.20 & $9,5(1,4)$ & $7,8(3,1)$ & 0.31 & $11,1(1,9)$ & $12,2(2,1)$ & 0,79 \\
$\mathrm{MAP}(\mathrm{mmHg})$ & $70,8(10)$ & $70,1(18)$ & 0,92 & $74,0(13)$ & $67,5(12)$ & 0,51 & $71,2(21) 69,4(18)$ & 0,91 \\
$\mathrm{Cl}\left(/ / \mathrm{min} / \mathrm{m}^{2}\right)$ & $2,6(0,8)$ & $3,7(1,1)$ & $0,04^{*}$ & $2,8(1,4)$ & $3,8(1,7)$ & 0,39 & $3,5(1,2)$ & $4,5(1,5)$ & 0,21 \\
\hline $\mathrm{R}$ - Responder; NR- Non-responder; ${ }^{*} \mathrm{P}<0,05$ & & & & & &
\end{tabular}

\section{Conclusion:}

SVV index was shown to be a reliable predictor of fluid responsiveness during OLT. Further studies are warranted to elucidate the role of this and other dynamic indexes in the setting of liver transplantation.

\section{References:}

I. Shin $\mathrm{YH}$, et al. Utility of uncalibrated femoral stroke volume variation as a predictor of fluid responsiveness during the anhepatic phase of liver transplantation. Liver Transpl 20 I I; 17 : 53-59.

2. Biais $M$, et al. Uncalibrated pulse contour-derived stroke volume variation predicts fluid responsiveness in mechanically ventilated patients undergoing liver transplantation. Brit J Anaesth 2008; 101: 761-768. 